\title{
Generalized Plane Waves and Waveguide Modes in a Moving Isotropic Medium
}

\author{
Aalund, Mogens; Johannsen, Günther
}

Published in:

Journal of Applied Physics

Link to article, DOI:

$10.1063 / 1.1660605$

Publication date:

1971

Document Version

Publisher's PDF, also known as Version of record

Link back to DTU Orbit

Citation (APA):

Aalund, M., \& Johannsen, G. (1971). Generalized Plane Waves and Waveguide Modes in a Moving Isotropic Medium. Journal of Applied Physics, 42(7), 2669-2673. https://doi.org/10.1063/1.1660605

\section{General rights}

Copyright and moral rights for the publications made accessible in the public portal are retained by the authors and/or other copyright owners and it is a condition of accessing publications that users recognise and abide by the legal requirements associated with these rights.

- Users may download and print one copy of any publication from the public portal for the purpose of private study or research.

- You may not further distribute the material or use it for any profit-making activity or commercial gain

- You may freely distribute the URL identifying the publication in the public portal

If you believe that this document breaches copyright please contact us providing details, and we will remove access to the work immediately and investigate your claim 
The differential equation for $\tilde{E}(z)$ [see Eq. (15)] now becomes

$$
\left(\partial^{2} \tilde{E} / \partial z^{2}\right)+j\left\{\left[\left(\omega+\omega_{c}\right) / v_{z}\right]-\beta_{0}\right\}(\partial \widetilde{E} / \partial z)-\alpha_{0}^{2} \tilde{E}=0,
$$

where $\alpha_{0}^{2}$ is given by Eq. (23), with the appropriate $\beta_{0}[\mathrm{Eq}$. (A6)].

The final differential equation for the normalized field is exactly the same as for the synchronous case, [Eq. (26)], except that now

$$
\delta=\frac{1}{2}\left[\frac{\beta_{0}-\left(\omega+\omega_{c}\right) / v_{0}}{\alpha_{0}}\right]
$$

and

$$
Q=\left(\beta_{0} / \alpha_{0}\right)\left[\left(1+\omega_{c} / \omega\right) / 8 V_{0} I_{0}\right] P_{\text {in }} .
$$

From Eq. (A10), we see that with cyclotron wave in- teractions, the maximum output power and electronic efficiency are reduced by a factor of $\left(1+\omega_{c} / \omega\right)^{-1}$ compared to the synchronous wave case. Physically, this reduction arises because a larger fraction of the circuit power goes into transverse kinetic energy of the electrons in the cyclotron wave case.

* This work was done at Microwave Associates, Inc., Burlington, Mass, under U.S. Army Flectronics Laboratory Contract.

$\dagger$ Consultant to Microwave Associates, Inc.

1 A. E. Siegman, J. Appl. Phys. 31, 17 (1960)

2 T. Wessel-Berg, J. Elec. Control 14, 137 (1963).

${ }^{3} \mathrm{~J}$. E. Carroll, Proceedings of the Fifth International Microwave Tube Congress (Academic, New York, 1965), pp. 69-72.

${ }^{4}$ R. J. Briggs, S. F. Paik, and A. H. Gottfried (unpublished).

5. E. Rowe, Nonlinear Electron-Wave Interaction Phenomena (Academic, New York, 1965).

${ }^{6}$ R. J. Briggs, S. F. Paik, and A. H. Gottfried, Eighth International Conference on Microtuave and Optical Generation and Amplification (to be published).

\title{
Generalized Plane Waves and Waveguide Modes in a Moving Isotropic Medium
}

\author{
Mogens Aalund and Günther Johannsen \\ Danish Engineering Acadeny, Electrical Department, The Technical University of Denmark, Lyngby and Aalborg, Denmark
}

(Received 30 October 1970)

\begin{abstract}
The Lorentz transformation of plane-wave-like solutions and general waveguide modes is analyzed. A propagation and attenuation tensor is introduced. General Doppler equations and invariant phase quantities are shown to be simple consequences of the formalism. The concept of dispersion is discussed and a covariant condition connected with this concept is given. Covariant wave equations and dispersion relations are derived in a simple manner. The dispersion relations are used to analyze some special waveguide solutions including cutoff phenomena.
\end{abstract}

\section{INTRODUCTION}

This paper is concerned with the Lorentz transformation of certain solutions of Maxwell-Minkowski's equations including space-time attenuated plane waves and waveguide modes, the medium being homogeneous, isotropic, conducting, dispersive, and in uniform motion.

Since we are dealing with a single medium we may be guided by the basic idea that "all" problems are solved in the rest system of the medium [even in the case of usual waveguides because the relative motion between waveguide wall and medium does not effect the boundary conditions (consult Ref. 1)]. The task left is, therefore, to transform and reinterpret known results. The authors feel that this aspect has not been emphasized strongly enough in recent papers on the subject. ${ }^{2-8}$

We choose 4-dimensional tensor formalism which seems to be much more suitable for the purpose than traditional 3-dimensional vector formalism.

By a tensor we understand a tensor defined on the Lorentz transformation group. Latin subscripts run from 1 to 4 , Greek subscripts run from 1 to 3 . The coordinate $x_{t}=i c t$, where $t$ is the time and $c$ the speed of light in vacuum; therefore the metric tensor in 4 space is equal to the Kronecker symbol $\delta_{i j}$ (when cartesian spatial coordinates are used) and we do not distinguish between contravariant and covariant tensors. Repeated subscripts obey the summation convention, and commas in subscripts denote partial differentiation with respect to coordinates (or covariant differentiation since the metric tensor is independent of the coordinates).

\section{PLANE-WAVE-LIKE SOLUTIONS}

Consider the transformation matrix $a_{m n}$ for a proper Lorentz transformation, ${ }^{9}$ i.e., $x_{n}=a_{r n} x_{r}{ }^{*}, x_{n}{ }^{*}=a_{n r} x_{r}$. Without loss of generality we choose

$$
a_{m n}=\left(\begin{array}{cccc}
1 & 0 & 0 & 0 \\
0 & 1 & 0 & 0 \\
0 & 0 & \gamma & i \beta \gamma \\
0 & 0 & -i \beta \gamma & \gamma
\end{array}\right),
$$


FIG. 1. Motion of $K^{*}$ relative to $K$.

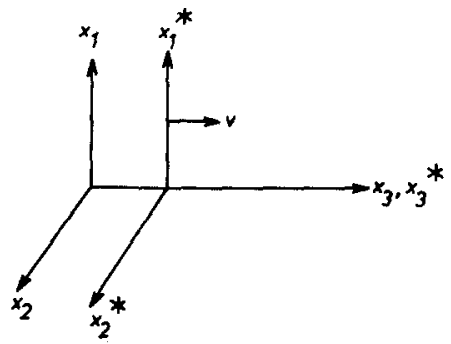

where $\gamma \equiv\left(1-\beta^{2}\right)^{-1 / 2}, \beta \equiv v / c$ and $v$ is the velocity of $K^{*}$ relative to $K$; the direction of $v$ is given in Fig. 1 .

Let $k_{n}$ be a constant (i.e., independent of $x_{r}$ ) tensor of first order, and let $f_{m n}, h_{m n}$ be antisymmetric constant tensors of second order. From these tensors we define the antisymmetric tensorfields

$$
\begin{aligned}
& f_{m n} \exp \left(i k_{r} x_{r}\right) \\
& h_{m n} \exp \left(i k_{r} x_{r}\right) .
\end{aligned}
$$

In Minkowski's theory the electromagnetic field in a moving medium can be described by means of two antisymmetric tensors of second order ${ }^{1}$ :

$$
\begin{gathered}
F_{m n}=\left(\begin{array}{cccc}
0 & c B_{3} & -c B_{2} & -i E_{1} \\
-c B_{3} & 0 & c B_{1} & -i E_{2} \\
c B_{2} & -c B_{1} & 0 & -i E_{3} \\
i E_{1} & i E_{2} & i E_{3} & 0
\end{array}\right) \\
\tilde{F}_{m n}=\left(\begin{array}{cccc}
0 & -i E_{3} & i E_{2} & c B_{1} \\
i E_{3} & 0 & -i E_{1} & c B_{2} \\
-i E_{2} & i E_{1} & 0 & c B_{3} \\
-c B_{1} & -c B_{2} & -c B_{3} & 0
\end{array}\right) \\
H_{m n}=\left(\begin{array}{cccc}
0 & H_{3} & -H_{2} & -i c D_{1} \\
-H_{3} & 0 & H_{1} & -i c D_{2} \\
H_{2} & -H_{1} & 0 & -i c D_{3} \\
i c D_{1} & i c D_{2} & i c D_{3} & 0
\end{array}\right), \\
\tilde{H}_{m n}=\left(\begin{array}{cccc}
0 & -i c D_{3} & i c D_{2} & H_{1} \\
i c D_{3} & 0 & -i c D_{1} & H_{2} \\
-i c D_{2} & i c D_{1} & 0 & H_{3} \\
-H_{1} & -H_{2} & -H_{3} & 0
\end{array}\right)
\end{gathered}
$$

where the dual tensors are given by $\widetilde{F}_{m n} \equiv(1 / 2 !) \epsilon_{m n r s} F_{r s}$, for example. ${ }^{10}$

It is well known that for a homogeneous, isotropic, dispersive, and conducting medium without sources there exist solutions of Maxwells equations which in the rest system $K^{\prime}$ of the medium are of the form as given by (2).

Because we are dealing with tensors these solutions are in any system of inertia given by

$$
\begin{aligned}
& F_{m n}=f_{m n} \exp \left(i k_{r} x_{r}\right) \\
& H_{m n}=h_{m n} \exp \left(i k_{r} x_{r}\right)
\end{aligned}
$$

which we call PWL solutions. $k_{r}$ are complex numbers.

\section{SOME TRANSFORMATION PROPERTIES}

Any tensor can be split into a sum of two tensors. We choose two tensors $\lambda_{n}$ and $\alpha_{n}$ so that

$$
k_{n}=\lambda_{n}+i \alpha_{n}
$$

and define $\lambda_{\nu} \equiv \operatorname{Re}\left(k_{\nu}\right), \quad \lambda_{4} \equiv i \operatorname{Im}\left(k_{4}\right), \quad \alpha_{\nu} \equiv \operatorname{Im}\left(k_{\nu}\right)$, $\alpha_{4} \equiv-i \operatorname{Re}\left(k_{4}\right)$. This definition is consistent (i.e., independent of the inertial system) which is readily seen from (1).

We call $\lambda_{n}$ propagation tensor and $\alpha_{n}$ attenuation tensor and the quantity

$$
\omega \equiv-i c \lambda_{4}
$$

is the frequency.

Depending on the values of $\lambda_{n}$ and $\alpha_{n}$ (4) can be interpreted as a (non)homogeneous plane wave, (non)attenuated in some/all coordinates or (4) cannot be interpreted as a travelling wave at all. The interpretation depends on the inertial system as discussed in Ref. 8 and is in principle settled by the fact that $\lambda_{n}$ and $\alpha_{n}$ are tensors.

The relativistic Doppler equations ${ }^{8}$ are given by $\lambda_{n}, \alpha_{n}$ and the tensor transformation law:

$$
\begin{aligned}
\lambda_{n}=a_{r n} \lambda_{r}{ }^{*}, & \lambda_{n}{ }^{*}=a_{n r} \lambda_{r} \\
\alpha_{n}=a_{r n} \alpha_{r}{ }^{*}, & \alpha_{n}{ }^{*}=a_{n r} \alpha_{r} .
\end{aligned}
$$

If $\lambda_{v}$ is proportional with $\alpha_{v}$ (i.e., the wave is homogeneous) in one system of inertia this is obviously in general not the case in another intertial system. (Of course, if $\lambda_{n}$ is proportional with $\alpha_{n}$ this proportionality is independent of the inertial frame.)

The tensor product

$$
\Phi \equiv k_{n} x_{n}
$$

is invariant, and substituting (5) into (8) we get the invariants

$$
\begin{aligned}
\operatorname{Re}(\Phi) & =\lambda_{n} x_{n} \\
\operatorname{Im}(\Phi) & =\alpha_{n} x_{n} .
\end{aligned}
$$

If $\lambda_{\nu} \lambda_{\nu} \neq 0\left(\alpha_{\nu} \alpha_{\nu} \neq 0\right)$, (9) can be used to define a propagation (attenuation) phase plan and velocity, e.g., $v_{p}=-i c \lambda_{4} /\left(\lambda_{\nu} \lambda_{v}\right)^{1 / 2}=\omega /\left(\lambda_{v} \lambda_{v}\right)^{1 / 2}$.

Similarily with (5) the tensors $f_{m n}, h_{m n}$ can be split into two tensors:

$$
\begin{aligned}
& f_{m n}=a_{m n}+i b_{m n} \\
& h_{m n}=d_{m n}+i e_{m n},
\end{aligned}
$$

where the quantities on the right sides are antisym- 
metric tensors given by $a_{\mu \nu} \equiv \operatorname{Re}\left(f_{\mu \nu}\right), a_{m 4} \equiv i \operatorname{Im}\left(f_{m 4}\right)$, $b_{\mu \nu} \equiv \operatorname{Im}\left(f_{\mu \nu}\right), b_{m 4} \equiv-i \operatorname{Re}\left(f_{m 4}\right) .\left(d_{m n}, e_{m n}\right.$ are defined in quite the same way by means of $h_{m n}$.) Again this definition is seen to be consistent.

Phase angles $\phi_{(m n)}$ may be defined by

$$
\begin{gathered}
\tan \phi_{(m n)} \equiv b_{m n} / a_{m n} \\
\tan \psi_{(m n)} \equiv e_{m n} / d_{m n} . \quad \text { (no summation) }
\end{gathered}
$$

It is noticed that phase angles (or differences between two of them) in general are not Lorentz invariants as pointed out in Ref. 8 but they are readily found by means of (11) in any system of inertia.

A PWL solution is easily transformed from one system of inertia to another. By inspection of the transformation matrix (1) we conclude that an element of any tensor (of arbitrary order) is uneffected by the Lorentz transformation if none of the suffix values exceed the integer 2, e.g., $k_{2}{ }^{*}=k_{2}, h_{12}{ }^{*}=h_{12}$.

Furthermore, if a tensor $T_{m n}$ is antisymmetric then $T_{34}{ }^{*}=T_{34}$ [and therefore also $T_{43}{ }^{*}=T_{43}$ because (anti)symmetry is an invariant property], for

$$
\begin{aligned}
T_{34}{ }^{*}=a_{3 r} a_{48} T_{r s}=a_{33} a_{48} T_{38}+a_{34} a_{4 s} T_{4 s} \\
\quad=a_{33} a_{44} T_{34}+a_{34} a_{43} T_{43}=T_{34}\left(a_{33} a_{44}-a_{34} a_{43}\right)=T_{34}
\end{aligned}
$$

because $\operatorname{det}\left(a_{r s}\right)=1$.

Applying these statements to the field tensors (3) we see that all field components with $x_{3}$ direction are Lorentz invariant (as it should be).

Finally it is noticed that due to the zeroes in (1) the amount of calculation work is considerably reduced when a tensor is to be transformed.

\section{DISPERSION}

The concept of dispersion is usually based on the assumption that the field can be decomposed into timeharmonic components in the rest system $K^{\prime}$ of the medium. Such a component can be written as

$$
g^{\prime}\left(x_{\nu}{ }^{\prime}\right) \exp \left(i l_{4}{ }^{\prime} x_{4}{ }^{\prime}\right)
$$

where $l_{4}{ }^{\prime}$ is a constant and $g^{\prime}$ is a function independent of the time coordinate $x_{4}{ }^{\prime}$. The frequency is given by $\omega^{\prime} \equiv-i c l_{4}^{\prime}\left[\right.$ cf. (6)]. Furthermore $\omega^{\prime}$ has to be real, i.e.,

$$
\operatorname{Im}\left(\omega^{\prime}\right)=\operatorname{Re}\left(l_{4}^{\prime}\right)=0 .
$$

PWL solutions as given by (4) are obviously time harmonic in $K^{\prime}$ if

$$
\alpha_{4}{ }^{\prime}=0 .
$$

This equation can be translated into covariant tensor language. To do this we make use of the velocity tensor $U_{n}$ of an inertial system $K$ with respect to the rest system $K^{\prime}$ which in $K^{\prime}$ is given by ${ }^{9}$

$$
U_{n}{ }^{\prime}=(0,0,0, i c) \text {. }
$$

Since $\alpha_{4}{ }^{\prime}=\alpha_{r}{ }^{\prime} U_{r}{ }^{\prime} / i c=\alpha_{r} U_{r} / i c$, (14) may be written as

$$
\alpha_{\tau} U_{r}=0 \text {. }
$$

By means of (6) and (15) we derive $\omega^{\prime}=-\lambda_{4}{ }^{\prime} U_{4}{ }^{\prime}=$ $-\lambda_{r}^{\prime} U_{r}^{\prime}$, i.e.,

$$
\omega^{\prime}=-\lambda_{r} U_{r} .
$$

If the PWL solution is time harmonic in $K^{\prime}$ we get from (5), (16), and (17) that

$$
\operatorname{Im}\left(k_{r} U_{r}\right)=0 \text {, }
$$

which is equivalent with the condition (14) or (16), and that the frequency in $K^{\prime}$ is given by

$$
\omega^{\prime}=-k_{r} U_{r} .
$$

In Minkowski's theory the constitutive parameters are invariant quantities, i.e., in the case of a dispersive medium $\epsilon^{\prime}\left(\omega^{\prime}\right)=\epsilon\left(\omega^{\prime}\right)$, say. Using (17) or (19) this function may be written with covariant argument as $\epsilon\left(-\lambda_{r} U_{r}\right)$ or $\epsilon\left(-k_{r} U_{r}\right)$.

Though a PWL solution remains a PWL solution under a Lorentz transformation the condition $\alpha_{4}=0$ (time harmonic in $K$ ) alone does not imply $\alpha_{4}{ }^{*}=0$ in another inertial system. In fact only if $\alpha_{3}=\alpha_{4}=0$ (no attenuation in the $x_{3}$ and $x_{4}$ coordinate) then $\alpha_{3}{ }^{*}=$ $\alpha_{4}{ }^{*}=0$ as seen by inspection of (1).

Consider a PWL "solution" with $\alpha_{4}=0, \alpha_{3} \neq 0$ in $K$ different from $K^{\prime}$, i.e., $\alpha_{4}^{\prime} \neq 0$. If we are working with a dispersion model as described above we have to show that the "solution" can be decomposed into timeharmonic components in $K^{\prime}$. This leads to convergency problems. Obviously the "solution" exists if we work with a dispersion model as suggested in Ref. 8, where $\epsilon$ (for example) is a function of the complex variable $-i c k_{4}{ }^{\prime}=-i c\left(\lambda_{4}{ }^{\prime}+i \alpha_{4}{ }^{\prime}\right)=-k_{r} U_{r}$, because condition (14), (16), (18) is no longer necessary, but the choice of a dispersion model is not only a question of mathematical convenience.

\section{THE WAVE EQUATION}

Not every set of tensorfields (2) represents a PWL solution; the Maxwell-Minkowski's equations have to be satisfied.

In the rest system $K^{\prime}$ of the medium the well-known condition ("dispersion relation")

$$
k_{\nu}{ }^{\prime} k_{\nu}{ }^{\prime}+n^{2} k_{4}{ }^{2}-\sigma \mu c k_{4}{ }^{\prime}=0
$$

is obtained by substituting (2) into the wave equation

$$
\left(\nabla^{\prime 2}+n^{2} \frac{\partial^{2}}{\partial x_{4}{ }^{2}}-i c \sigma \mu \frac{\partial}{\partial x_{4}^{\prime}}\right)\left\{\begin{array}{l}
F_{m n}^{\prime} \\
H_{m n}{ }^{\prime}
\end{array}=0 .\right.
$$

In order to get covariant formulations of $(20)$ and (21) one may derive the tensor wave equation in $K$ from the tensor formulation of Maxwell-Minkowski's equations. Another way is to translate (21) directly into tensor language which will be done here.

From (21) we get

$$
T_{, m m}{ }^{\prime}+\left(n^{2}-1\right) T_{, 44^{\prime}}-i c \sigma \mu T_{, 4}{ }^{\prime}=0,
$$

which by means of (15) can be written as

$$
T_{, m m}-\kappa T_{, m n} U_{m} U_{n}-\sigma \mu T_{, m} U_{m}=0,
$$


where $T$ stands for a field tensor and

$$
\kappa \equiv\left(n^{2}-1\right) / c^{2} .
$$

We have omitted the primes in (23) because it is a tensor equation.

In a similar way or by inserting (4) into (23) we get the condition

$$
\Lambda=0,
$$

where

$$
\Lambda \equiv k_{m} k_{m}-\kappa\left(k_{m} U_{m}\right)^{2}+i \sigma \mu\left(k_{m} U_{m}\right) .
$$

Equation (25) ensures that (2) is a solution of (23).

From (5) and (26) we finally derive the real invariants

$$
\begin{array}{r}
\operatorname{Re}(\Lambda)=\lambda_{m} \lambda_{m}-\alpha_{m} \alpha_{m}-\kappa\left[\left(\lambda_{m} U_{m}\right)^{2}-\left(\alpha_{m} U_{m}\right)^{2}\right]-\sigma \mu \alpha_{m} U_{m} \\
\operatorname{Im}(\Lambda)=2 \lambda_{m} \alpha_{m}-2 \kappa \lambda_{m} U_{m} \alpha_{n} U_{n}+\sigma \mu \lambda_{m} U_{m} .
\end{array}
$$

\section{GENERALIZED PLANE-WAVE-LIKE SOLUTIONS}

PWL solutions as given by (4) are not very useful if we are concerned with waveguides containing a moving medium. To this purpose we will look at field solutions which can be written in the form

$$
\begin{aligned}
& F_{m n}=f_{m n}\left(x_{1}, x_{2}\right) \exp \left(i k_{r} x_{r}\right) \\
& H_{m n}=h_{m n}\left(x_{1}, x_{2}\right) \exp \left(i k_{r} x_{r}\right), \quad k_{1}=k_{2}=0,
\end{aligned}
$$

where $f_{m n}, h_{m n}$ are tensorfields independent of $x_{3}, x_{4}$ and $k_{r}$ is a constant tensor with zero first and second coordinate. From the remarks made in Secs. II and III it becomes evident that this definition is consistent.

The results from Secs. III and IV may be extended to solutions as given by (28), in particular the tensor $k_{r}$ can be split into two parts as defined by (5) (with $\lambda_{1}=\lambda_{2}=\alpha_{1}=\alpha_{2}=0$ ).

Condition (25) in Sec. V must be generalized. Substituting (28) into (23) leads to the 2-dimensional wave equation

$$
\left(\nabla_{t}^{2}-\Lambda\right)\left\{\begin{array}{l}
f_{m n}\left(x_{1}, x_{2}\right) \\
h_{m n}\left(x_{1}, x_{2}\right)
\end{array}=0,\right.
$$

where the invariant $\Lambda$ is still given by (26) and $\nabla_{t}^{2} \equiv$ $\left(\partial^{2} / \partial x_{1}{ }^{2}\right)+\left(\partial^{2} / \partial x_{2}{ }^{2}\right)$ is covariant under a Lorentz transformation given by (1).

\section{WAVEGUIDES}

Consider a waveguide parallel to the $x_{3}$ direction with constant (i.e., independent of $x_{3}$ ) cross section and containing a uniformly moving, isotropic medium.

As in Sec. II our point of departure is the rest system $K^{\prime}$ of the medium where "all" problems are solved. We do this because the 2-dimensional wave equation (29) is valid in every system of inertia and the boundary conditions are not effected by the motion of the waveguide walls. ${ }^{1}$
In $K^{\prime}$ we know that solutions as given by (28) exist. These solutions are usually classified as $T E$ and $T M$ modes. Since $k_{r}{ }^{\prime} x_{r}{ }^{\prime}=k_{r} x_{r}$ all these modes can readily be transformed to another inertial system as outlined in Sec. III. Some special transformation properties of the solutions under consideration may be worthwhile mentioning here:

Since $E_{3}{ }^{\prime}=E_{3}\left(D_{3}{ }^{\prime}=D_{3}\right), H_{3}{ }^{\prime}=H_{3}\left(B_{3}{ }^{\prime}=B_{3}\right)$ the concept of $T E$ and $T M$ waves is seen to be invariant.

Consider $T E$ modes, i.e., $E_{3}=0$ or $\widetilde{F}_{12}=0$ [cf. (3a)]. It follows from the Maxwell equations ${ }^{1,9}$

$$
\tilde{F}_{m n, n}=0
$$

in connection with (28) that

$$
\begin{aligned}
& \tilde{f}_{14}=-\left(k_{3} / k_{4}\right) \tilde{f}_{13} \\
& \tilde{f}_{24}=-\left(k_{3} / k_{4}\right) \tilde{f}_{23}
\end{aligned}
$$

in any inertial system.

From (31) and the transformation matrix (1) we easily obtain

where

$$
\begin{aligned}
& \tilde{f}_{13}=\alpha^{\prime} \tilde{f}_{13}{ }^{\prime} \\
& \tilde{f}_{23}=\alpha^{\prime} \tilde{f}_{23}{ }^{\prime},
\end{aligned}
$$

Furthermore (1) shows that

$$
k_{4}=\alpha^{\prime} k_{4}{ }^{\prime} .
$$

It is well known that in $K^{\prime}$ all field components can be expressed by $\tilde{f}_{34^{\prime}}$ (corresponding to $B_{3}{ }^{\prime}$ ). As to $E_{1}^{\prime}, E_{2}^{\prime}$ we have

$$
\begin{aligned}
& i \Lambda \tilde{f}_{23}{ }^{\prime}=k_{4}{ }^{\prime} \tilde{f}_{34,2^{\prime}} \\
& i \Lambda \tilde{f}_{13}{ }^{\prime}=k_{4}{ }^{\prime} \tilde{f}_{34,1^{\prime}} \text {. }
\end{aligned}
$$

The transformation of (35) is now trivial. Obviously $\tilde{f}_{34}{ }^{\prime}\left(x_{1}{ }^{\prime}, x_{2}{ }^{\prime}\right)=\tilde{f}_{34}\left(x_{1}, x_{2}\right)$ for all $x_{1}{ }^{\prime}=x_{1}, x_{2}{ }^{\prime}=x_{2}$ (cf. Sec. VI) and by means of (32) and (34) we see that (35) are covariant equations (i.e., the primes can be omitted; this also holds for the equations expressing $E_{1}{ }^{\prime}, E_{2}{ }^{\prime}$ in terms of $B_{3}{ }^{\prime}$ because $k_{r} x_{r}$ is invariant).

From (31) we deduce that the equations expressing $B_{1}{ }^{\prime}, B_{2}{ }^{\prime}$ in terms of $B_{3}{ }^{\prime}$ are covariant.

As to $T M$ modes we finally conclude by means of symmetry considerations that the equations expressing $H_{1}{ }^{\prime}, H_{2}{ }^{\prime}, D_{1}{ }^{\prime}, D_{2}{ }^{\prime}$ in terms of $D_{3}{ }^{\prime}$ are covariant if $\sigma=0$.

\section{SPECIAL WAVEGUIDE SOLUTIONS, CUTOFF}

Consider a waveguide mode specified by the condition

$$
\Lambda=-\Gamma^{2},
$$

where the real mode number $\Gamma^{2}$ is determined by the covariant, 2-dimensional wave equation (29) in connection with the configuration of the waveguide cross section.

$\Lambda$ is given by (26) or (27), i.e., (36) represents two relations between the 4 nonvanishing components $\lambda_{3}$, 
$\lambda_{4}, \alpha_{3}$, and $\alpha_{4}$ of the propagation and attenuation tensor, necessary and sufficient conditions for the existence of a solution.

Solutions belonging to a given mode (i.e., $\Gamma^{2}$ ) including cutoff phenomena can readily be analyzed by means of these relations. We will do this for a nondispersive, lossless medium in two cases, the solutions being time harmonic in the rest system $K^{\prime}$ or in another inertial system $K$.

Case I: $\alpha_{4}{ }^{\prime}=0, \sigma=0$.

The transformation formula of the attenuation tensor is reduced to [cf. (1) and Fig. 1, $K^{\prime}$ coincident with $K^{*}$ ]

$$
\begin{aligned}
& \alpha_{4}=i \beta \alpha_{3} \\
& \alpha_{3}=\gamma \alpha_{3}{ }^{\prime} .
\end{aligned}
$$

Using (27), (6), and (15) condition (36) can be expressed by the well-known equations

$$
\begin{aligned}
\lambda_{3}{ }^{2}-\alpha_{3}{ }^{2} & =n^{2}\left(\omega^{\prime} / c\right)^{2}-\Gamma^{2} \\
\lambda_{3}{ }^{\prime} \alpha_{3}{ }^{\prime} & =0,
\end{aligned}
$$

and there is no need for analyzing the covariant edition of (38).

Above cutoff, i.e., when $\left(\omega^{\prime} / c\right)>(\Gamma / n)$ we obviously have $\alpha_{3}{ }^{\prime}=0$ and therefore $\alpha_{3}=\alpha_{4}=0$. The solutions are nonattenuated (harmonic) in the space and the time coordinate.

$\lambda_{3}{ }^{\prime}$ is given by (38a) with $\alpha_{3}{ }^{\prime}=0$ and may be positive or negative (corresponding to a wave travelling in the positive or negative $x_{3}{ }^{\prime}$ direction). Finally $\lambda_{3}$ and $\lambda_{4}$ are given by the tensor transformation law which can be written as

$$
\begin{aligned}
& \lambda_{3}=\gamma\left[\lambda_{3}{ }^{\prime}+\beta\left(\omega^{\prime} / c\right)\right] \\
& \omega / c=\gamma\left[\left(\omega^{\prime} / c\right)+\beta \lambda_{3}{ }^{\prime}\right] .
\end{aligned}
$$

From (39a) it is seen that if $\beta \lambda_{3}{ }^{\prime}<0$, then $\lambda_{3}=0$ is possible and the corresponding frequency in $K^{\prime}$ is given by $\omega^{\prime} / c=\Gamma /\left(n^{2}-\beta^{2}\right)^{1 / 2}(>\Gamma / n$, the cutoff frequency in $\left.K^{\prime}\right)$. The field is time harmonic but independent of $x_{3}$. This is a relativistic effect; the wave has been "run down" in such a way that a field component is in phase at all points in the $x_{3}$ direction. (Simultaneousness depends on the system of inertia!) One may call this effect cutoff, but the nature of this relativistic phenomenon is quite different from the well-known cutoff effect of a waveguide in the rest system $K^{\prime}$ of the medium.

$\omega=0 \quad$ when $\quad \omega^{\prime} c=|\beta| \Gamma /\left(n^{2} \beta^{2}-1\right)^{1 / 2}$ and $\beta \lambda_{3}{ }^{\prime}<0$ which is only possible when $(n \beta)^{2}>1$ (i.e., above the Cerenkov velocity as it should be).

Below cutoff in $K^{\prime}$, i.e., when $\omega^{\prime} / c<\Gamma / n$ we have $\lambda_{3}{ }^{\prime}=0$ so that (39) reduces to

$$
\begin{gathered}
\lambda_{3}=\gamma \beta\left(\omega^{\prime} / c\right) \\
\omega / c=\gamma\left(\omega^{\prime} / c\right) .
\end{gathered}
$$

In this case $\alpha_{3}^{\prime}$ is different from zero and given by (38a) implying that $\alpha_{3} \neq 0$ and $\alpha_{4} \neq 0$ [cf. (37)]. This means that the solutions represent space-time attenuated waves in $K$.

It is a relativistic effect that $\lambda_{3} \neq 0$ (in spite of $\lambda_{3}{ }^{\prime}=0$ ) and that $\alpha_{1} \neq 0$ (in spite of $\alpha_{4}{ }^{\prime}=0$ ). When the speed $v$ of $K$ relative to the medium becomes comparable with the speed of light in vacuum (i.e., $\beta^{2} \approx 1$ ) then $\left|\lambda_{3}\right| \approx$ $\omega / c$ and no cutoff effect is therefore observed in $K$.

Case $I I: \alpha_{4}=0, \sigma=0$.

We readily derive

and

$$
\begin{gathered}
\alpha_{4}{ }^{\prime}=-i \beta \alpha_{3}{ }^{\prime} \\
\alpha_{3}=(1 / \gamma) \alpha_{3}{ }^{\prime}
\end{gathered}
$$

$$
\begin{gathered}
\lambda_{3}{ }^{2}-n^{2}\left(\omega^{\prime} / c\right)^{2}-\alpha_{4}{ }^{\prime 2}\left[n^{2}-\left(1 / \beta^{2}\right)\right]+\Gamma^{2}=0 \\
\alpha_{3}\left[\lambda_{3}{ }^{\prime}+n^{2} \beta\left(\omega^{\prime} / c\right)\right]=0 .
\end{gathered}
$$

From (42b) we see that $\alpha_{3}{ }^{\prime}=0$ or

$$
\lambda_{3}^{\prime}=-n^{2} \beta\left(\omega^{\prime} / c\right) \text {. }
$$

Since $\alpha_{3}^{\prime}=0$ implies $\alpha_{3}=\alpha_{4}=0$ in all systems $K$ we already have treated this situation under case $I$.

Assuming that $\alpha_{3}{ }^{\prime} \neq 0$ we strictly speaking leave our basic idea as mentioned in the introduction. When $\beta^{2}$ is comparable with 1 we are not "familiar" with the problem of how to excite a solution which is time harmonic in $K$ (but not in $K^{\prime}$ ) whether the source is or is not at rest in $K^{\prime}$ ! Nevertheless we may discuss the equations.

Inserting (43) into (42a) leads to the equation

$$
-\left(\alpha_{4}{ }^{\prime}\right)^{2}=n^{2} \beta^{2}\left[\frac{(\Gamma / n)^{2}}{1-n^{2} \beta^{2}}-\left(\frac{\omega^{\prime}}{c}\right)^{2}\right] .
$$

Since $-\left(\alpha_{1}{ }^{\prime}\right)^{2}>0$ we get the conditions that $n^{2} \beta^{2}<1$ and $\omega^{\prime} / c<(\Gamma / n) /\left(1-n^{2} \beta^{2}\right)^{1 / 2}$. Finally from (39) and (42) we obtain

$$
\begin{aligned}
& \lambda_{3}=-\gamma \beta\left(n^{2}-1\right)\left(\omega^{\prime} / c\right) \\
& \omega / c=\gamma\left(1-n^{2} \beta^{2}\right)\left(\omega^{\prime} / c\right)
\end{aligned}
$$

which in turn leads to

$$
\lambda_{3}=-\beta\left(n^{2}-1\right) /\left(1-n^{2} \beta^{2}\right)(\omega / c) .
$$

${ }^{1}$ A. Sommerfeld, Electrodynamics (Academic, New York, 1967).

${ }^{2}$ J. R. Collier and C. T. Tai, IEEE Trans. Microwaves Theory Techniques 13, 441 (1965).

${ }^{3}$ L. J. Du and R. T. Compton, Jr., IEEE Trans. Microwaves Theory Techniques 14, 358 (1966).

4 T. Shiozawa, IEEE Proc. 54, 1984 (1966).

${ }^{5}$ H. Berger, IEEE Proc. 55, 1214 (1967).

6 P. Daly, IEEE Trans. Microwaves Theory Techniques 15, $274(1967)$.

${ }^{7}$ H. Berger and J. W. E. Griemsmann, IEEE Trans. Microwaves Theory Techniques 15,11 (1968).

${ }^{8}$ H. Berger and J. W. E. Griemsmann, J. Appl. Phys. 39, 3569 (1968).

${ }^{\circ} \mathrm{C}$. Møller, The Theory of Relativity (Oxford U. P., England, 1962).

${ }_{10} \mathrm{~J}$. L. Synge and A. Schild, Tensor Calculus (Toronto Press, Canada, 1962). 